'Laboratorio de Estrés y Salud. Doctorado en Psicología, Facultad de Educación, Ciencias Sociales y Humanidades, Universidad de La Frontera. Temuco, Chile. ${ }^{2}$ Departamento de Psicología, Facultad de Educación, Ciencias Sociales y Humanidades, Universidad de La Frontera. Temuco, Chile. ${ }^{3}$ Universidad de La Frontera, Programa de Formación de investigadores UFRO Postdoctorales, VRIP19P001. Temuco, Chile. aicenciado en Psicología. 'Psicólogo; Doctor en Psicología. 'Psicólogo; Doctor (Ph.D) en Psicología de la Salud.

dEstudiante de Doctorado en Psicología.

Esta investigación fue financiada por CONICYT (Comisión Nacional de Investigación Científica y Tecnológica, Gobierno de Chile), en forma total por el proyecto FONDECYT POSTDOCTORADO N³180534 cuya investigadora responsable es la Dra.

Margarita Cancino y parcialmente por el proyecto FONDECYT REGULAR 1180463 cuyo investigador principal es el Dr. Manuel S. Ortiz.

Los autores declaran no tener conflictos de interés.

Recibido el 4 de enero 2021, aceptado el 12 de mayo de 2021.

Correspondencia a: Margarita Cancino Departamento de Psicología, Universidad de La Frontera. Montevideo 0830. Temuco, Chile. margarita.cancino@ufrontera.cl

\section{Factores de riesgo cardiometabólico y funcionamiento cognitivo: el rol de la reserva cognitiva}

\author{
LUCIANA SEPÚLVEDA-PEZOA ${ }^{1,2, a}$, DANIELA GÓMEZ-PÉREZ2,3,b, \\ MANUEL S. ORTIZ ${ }^{1,2, c}$, BELÉN SALINAS-REHBEIN ${ }^{1,2, d}$, \\ MARGARITA CANCINO ${ }^{2, b}$
}

\section{Association between cardiometabolic risk and cognitive impairment}

Background: Several risk factors are associated with cognitive impairment in older people, but little attention has been paid to cardiometabolic variables, as well as how cognitive reserve can mediate this association. Aim: To determine the association of cardiometabolic risk factors with cognitive functioning and whether the cognitive reserve mediates this association. Material and Methods: A cross-sectional study with 300 participants with a median age of 56 years (53\% male) was conducted. Participants had a cognitive functioning assessment. Fasting glucose, HDL-cholesterol, and blood pressure were measured. Results: A Structural Equation Modeling, revealed a direct effect of cardiometabolic latent risk factors on both a cognitive functioning ( $\beta=-.204 ; p=.056)$, and cognitive reserve factors $(\beta=-.236, p=.04)$. Cognitive reserve was directly associated with cognitive functioning $(\beta=.327, p=.01)$, but did not mediate the association between the cardiometabolic risk factors and cognitive functioning $(\beta=.077, p=.07)$. The overall goodness of fitness was excellent $\chi^{2}(50)=58.357, p=.195$, comparative fit index $=.980$, Tucker-Lewis Index $=.974$, root mean square error of approximation $=.023$ and Standardized Root Mean Square Residual $=.041$. Conclusions: $A$ direct effect of cardiometabolic risk factors on cognitive functioning was observed.

(Rev Med Chile 2021; 149: 1134-1140)

Key words: Aging; Cardiometabolic Risk Factors; Cognition; Cognitive Reserve.
L a población mundial está envejeciendo ${ }^{1} \mathrm{y}$ Chile no es la excepción. La Encuesta de Caracterización Socioeconómica Nacional $(\mathrm{CASEN})^{2}$ reportó que la población mayor a 45 años se ha incrementado en 16 puntos porcentuales entre los años 1990 y 2017; de acuerdo al último Censo $2017^{2}$, los individuos mayores a 50 años corresponden a $28 \%$ de la población, de los cuales $54 \%$ son mujeres. Se espera que para el año 2050 este grupo etario constituya $44 \%$ de la población chilena ${ }^{3}$, mientras la proyección a partir del último Censo, indica que las personas mayores de 50 años representarán $46 \%$ de la población ${ }^{2}$.

El envejecimiento es un proceso heterogéneo natural, cuyas variaciones son producto de experiencias a lo largo de la vida y que ocurren por influencia del entorno y/o del comportamiento adquirido por la persona ${ }^{4}$, impactando a nivel físico, social y psicológico ${ }^{5}$. El proceso de envejecer aumenta el riesgo de desarrollar distintas 
enfermedades ${ }^{6}$, como diabetes mellitus tipo 2 (DM2) e hipertensión arterial ${ }^{7}$. Asimismo, ha sido vinculado con cambios cerebrales e impacto en el funcionamiento cognitivo (FC) $)^{8}$, y en las habilidades de resolución de problemas, razonamiento, aprendizaje y atención ${ }^{9}$. De esta forma, el envejecimiento se asocia con problemas de memoria, cambios en la velocidad de procesamiento, enlentecimiento motor, menor fluidez verbal y mayor rigidez cognitiva, aunque el lenguaje y la memoria semántica tienden a ser más resistentes al paso del tiempo ${ }^{10}$.

Aunque el declive en el FC normal se asocia al envejecimiento, hay diversos factores de riesgo que se relacionan a este deterioro. De ellos, en los últimos años se ha prestado mayor atención al rol que los factores cardiometabólicos tienen en la alteración del FC. Es más, estos factores tienen un rol importante en la posibilidad de desarrollar demencia en edades posteriores ${ }^{11}$. Por ejemplo, la acumulación de alteraciones de marcadores biológicos, tales como elevación de presión arterial, dislipidemias y alteración de glucosa sanguínea, se asocia con deterioro de la función ejecutiva, velocidad del procesamiento y memoria verbal ${ }^{12}$. A su vez, la hipertensión arterial está relacionada con una disminución de la función cognitiva ${ }^{13}$, pudiendo ser un factor predictor de demencia y/o deterioro cognitivo, lo cual ha sido demostrado en un estudio transversal con 500 participantes, donde valores altos de presión sistólica se asociaron con un peor rendimiento en pruebas de memoria y función ejecutiva ${ }^{14}$. Además, las intervenciones destinadas a disminuir la presión arterial con medicamentos en personas más jóvenes permiten reducir el riesgo de demencia en edades más avanzadas ${ }^{15}$, así como también atenuar el riesgo de deterioro cognitivo en personas sanas ${ }^{16}$.

Otro factor de riesgo cardiometabólico es el colesterol alterado, el cual en edades avanzadas desempeña un rol importante en el desarrollo de deterioro cognitivo y Enfermedad de Alzheimer $(\mathrm{EA})^{17}$. Específicamente, el colesterol HDL es un factor que contribuye a las diferencias individuales en el funcionamiento cognitivo de los adultos mayores $^{18}$, y se ha observado que niveles bajos de colesterol HDL pueden provocar alteraciones en la memoria ${ }^{19}$. En la misma línea, un estudio longitudinal de 6 años con 1.003 adultos, demostró un mejor rendimiento de memoria en aquellas perso- nas con altos niveles de colesterol HDL en sangre ${ }^{20}$.

A las variables antes mencionadas, se debe añadir la diabetes mellitus (DM), la cual es un factor de riesgo para el desarrollo de enfermedad cardiovascular ${ }^{21}$, así como también para demencia tipo Alzheimer o vascular. La DM2 se asocia con cambios en el aprendizaje, memoria, flexibilidad y velocidad mental ${ }^{22}$, además de deterioro en la función ejecutiva ${ }^{23}$. Un estudio realizado con adultos mayores italianos, reportó que quienes fueron diagnosticados con DM tuvieron $80 \%$ de posibilidades de experimentar algún tipo de deterioro cognitivo ${ }^{24}$.

En consecuencia, los factores de riesgo cardiometabólico tienen un efecto directo en el funcionamiento cognitivo ${ }^{25}$, no obstante, es posible hipotetizar que la reserva cognitiva (RC), tiene un rol mediador en esta asociación ${ }^{26}$. La RC es definida como un mecanismo potencial que permite enfrentar daños a nivel cerebral constituyendo, por tanto, la capacidad de utilizar recursos cognitivos y usar vías alternativas para compensar los daños producto del deterioro cognitivo ${ }^{27}$. La RC se potencia a través de actividades que estimulan la función cognitiva ${ }^{28}$, tales como actividades laborales, de ocio, práctica de actividad física, entre otras. De esta forma, es esperable la existencia de una relación directa entre RC y FC. En efecto, un estudio realizado con adultos mayores chilenos evidenció esta asociación, demostrando un mejor rendimiento en tareas cognitivas en sujetos con alta $\mathrm{RC}^{29}$. Asimismo, una investigación transversal con datos del Newcastle 85+ Study, determinó una asociación positiva entre RC y la función cognitiva global y por dominios específicos de adultos mayores ${ }^{28}$.

Finalmente, es importante destacar que los factores de riesgo cardiometabólico, pueden afectar directamente la RC y de esta forma impactar en el FC. De estos factores cardiometabólicos, la presión arterial tiene un rol central. Un estudio con participantes de edades entre 53 y 94 años, determinó una relación inversa entre presión arterial elevada y reserva cognitiva ${ }^{30}$, mientras que otra investigación evidenció que la relación entre hipertensión arterial y memoria está mediada por el indicador de actividades cognitivas de ocio de la $\mathrm{RC}^{31}$.

En consecuencia, es posible hipotetizar que los factores de riesgo cardiometabólico tienen un efecto en el FC de los sujetos y la RC tendría un rol mediador en esta asociación. Por lo 
tanto, el objetivo de este estudio fue determinar la relación existente entre factores de riesgo cardiometabólico y el FC, y si esta relación está mediada por la RC.

\section{Método}

\section{Participantes}

Los participantes de este estudio están enrolados en el Proyecto FONDECYT de Postdoctorado No 3180534, el cual tiene un diseño no experimental, longitudinal de panel, de tres años de duración. A través de un muestro no probabilístico por conveniencia, se seleccionaron 300 sujetos entre 50 y 75 años de la región de La Araucanía, cuyo promedio de edad fue de 56,6 años (D.T. $=5,25)$, de los cuales 53\% son hombres. En relación a la escolaridad, $13 \%$ no ha completado la enseñanza media, $31 \%$ presenta estudios técnicos incompletos, $24 \%$ tiene estudios universitarios incompletos y $33 \%$ presenta un título universitario y/o de postgrado. El promedio de ingreso de los participantes fue de 749.188 pesos (D.T. $=41.708$ ). Los criterios de inclusión fueron no haber sufrido un accidente cerebrovascular en los últimos 5 años, estar libre de enfermedad cardiovascular y no estar bajo tratamiento anti-inflamatorio. En este estudio se consideraron los datos obtenidos en el primer año de ejecución del proyecto (2018).

\section{Instrumentos}

Funcionamiento cognitivo: Se utilizó la versión en español del test Addenbrooke's Cognitive Examination III (ACE-III) ${ }^{32}$, que incluye subescalas de atención, memoria, fluidez, lenguaje y habilidad visoespacial. El puntaje máximo es de 100 puntos. En este estudio se obtuvo una consistencia interna de 0,70 .

Reserva cognitiva: Se administró la Escala de Reserva Cognitiva ${ }^{33}$. Consta de 24 ítems, divididos en temáticas como formación-información, actividades de la vida diaria, hobbies-aficiones y vida social. Cada ítem cuenta con 5 opciones de respuesta: 1) nunca; 2) una o varias veces al año; 3) una o varias veces al mes; 4) siempre que me surge la oportunidad, y 5) tres veces por semana. Las preguntas están destinadas a indagar la frecuencia con que la persona ha realizado diferentes actividades en tres períodos del ciclo vital: a los 30 años, a los 50 y en la actualidad. Para efectos de estudio, se consideraron actividades realizadas actualmente. La consistencia interna fue de 0,83.

Factores de riesgo cardiometabólico: La concentración de glucosa se obtuvo a través del método de referencia de la hexoquinasa, mientras que la medición de colesterol HDL se determinó por medio de una medida directa/PEG. Se realizaron tres mediciones de presión arterial sistólica y diastólica con un equipo DINAMAP V100 (General Electric), de las que se utilizaron el promedio de la presión arterial sistólica de la segunda y tercera medida.

\section{Procedimiento}

Este estudio contó con la aprobación del Comité Ético Científico de la Universidad de La Frontera, cumpliendo con los principios éticos de la declaración de Helsinki. Todos los sujetos asistieron con ayuna de al menos ocho horas al Laboratorio de Estrés y Salud en donde la Investigadora Responsable hizo entrega del consentimiento informado en formato físico, el cual fue firmado por cada participante. Posteriormente, personal técnico tomó una muestra de sangre mediante un BD Vacutainer ${ }^{\circledR}$ UltraTouchTM Push Button Blood Collection Set. Adicionalmente, los participantes contestaron una encuesta formato online o papel según su preferencia, la cual contenía distintas escalas para medir variables sociodemográficas, psicológicas y conductuales. En un día diferente, estudiantes debidamente capacitadas, tomaron medidas de presión arterial y realizaron una evaluación cognitiva. Los sujetos fueron retribuidos económicamente con cinco mil pesos y obtuvieron los resultados de medidas antropométricas, presión arterial y perfil bioquímico.

\section{Plan de análisis}

Para el análisis de los datos se utilizó el software STATA en su versión 14.1, considerando un alfa nominal de 0,05 .

Las hipótesis de este estudio fueron probadas a través de Análisis de Ecuaciones.

Estructurales, estimando el efecto directo de los factores de riesgo cardiometabólico en el funcionamiento cognitivo y el efecto indirecto a través de reserva cognitiva. Además, se consideraron indicadores de bondad de ajuste convencionales: $\chi^{2}$ no significativo e Índice de ajuste comparativo $\mathrm{CFI} \geq 0,95, \mathrm{TLI} \geq 0,90$, Índice de ajuste basado en los residuos $S R M R \leq 0,08$ y RMSEA $\leq 0,06$. 


\section{Resultados}

Dado el incumplimiento del supuesto de normalidad multivariada (Mardias multivariate test $p \leq 0,001)$, los análisis se efectuaron con Método de Máxima Verosimilitud con corrección de Satorra-Bentler.

En este estudio se construyeron tres factores latentes (Figura 1), uno para cada variable de interés. El primero de ellos denominado Funcionamiento Cognitivo, estuvo compuesto por los indicadores de lenguaje $(\beta=0,648 ; p=0,000)$, habilidad visoespacial $(\beta=0,388 ; p=0,000)$, fluidez verbal $(\beta=0,613 ; p=0,000)$, atención $(\beta=0,400$; $p=0,000)$ y memoria $(\beta=0,492 ; p=0,000)$. El segundo identificado como Factores de Riesgo Cardiometabólico, que incluyó las variables observadas de presión arterial sistólica $(\beta=0,389$; $p=0,000)$, colesterol HDL $(\beta=-0,385 ; p=0,00)$ y glucosa $(\beta=0,414 ; p=0,02)$. Finalmente, el factor latente de Reserva Cognitiva, que incluyó los indicadores de vida diaria $(\beta=0,580 ; p=0,00)$, formación/información $(\beta=0,821 ; p=0,00)$, ocio $(\beta=0,647 ; p=0,00)$ y contacto social $(\beta=0,394$; $p=0,00)$. En todos estos factores latentes, las cargas factoriales $(\beta)$ de los indicadores fueron estadísticamente significativas y mayores a .3, resultado que indica una adecuada configuración de cada factor.

Respecto a los efectos directos entre las variables, es posible observar que los factores de riesgo cardiometabólico se asociaron directamente y de forma negativa con el FC $(\beta=-0,204, p=0,05)$, y con la RC $(\beta=-0,236, p=0,04)$, indicando que en presencia de mayor alteración de estos factores cardiometabólicos existe un menor FC y RC. Asimismo, la RC se asoció con mejor FC $(\beta=0,327$, $p=0,01)$ (Figura 1).

Respecto al mecanismo de mediación a través de la RC, este efecto no fue estadísticamente significativo $(\beta=0,077, p=0,07)$, sin embargo, el efecto total del modelo si lo fue $(\beta=0,281, p=0,04)$. Los

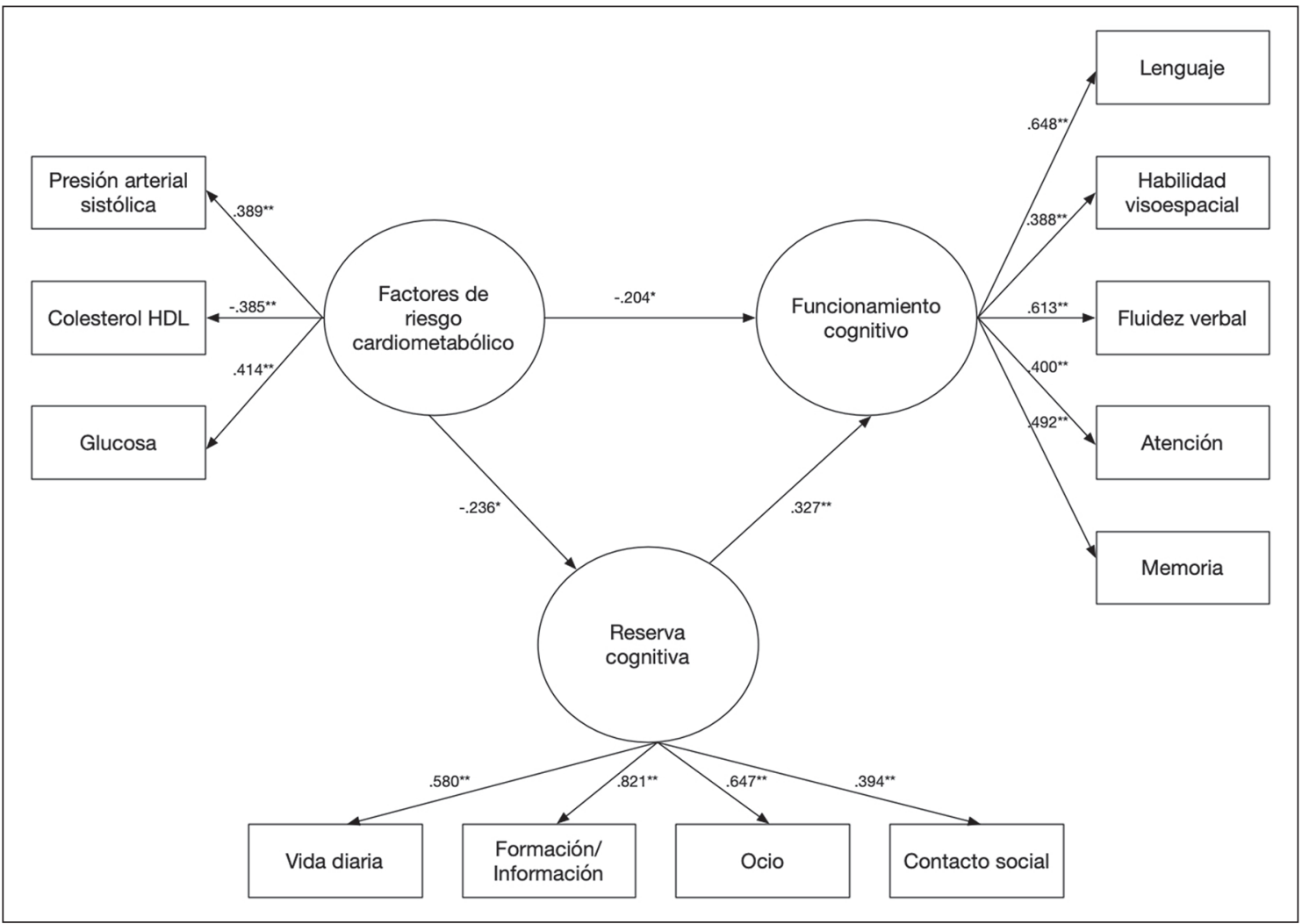

Figura 1. Modelo de relaciones entre factores de riesgo cardiometabólico y funcionamiento cognitivo en adultos. Fuente: elaboración propia. ${ }^{*} p \leq 0,05 ;{ }^{* *} p \leq 0,01$. 
indicadores de bondad de ajuste global del modelo fueron excelentes: $\chi^{2}{ }_{(50)}=58.357, p=0,195$, $\mathrm{CFI}=0,980$ TLI $=0,974$, RMSEA $=0,023$, SRMR $=0,041$ y el coeficiente de determinación fue 0,401 .

\section{Discusión}

Este estudio indagó la asociación entre los factores de riesgo cardiometabólico y el FC, así como también el efecto mediador de la RC. En primer lugar, se identificó una asociación entre los factores de riesgo cardiometabólico y el FC, lo cual indica que personas adultas que presentan elevados niveles de presión arterial y glucosa en sangre, y bajos niveles de colesterol HDL, tendrían un mayor declive en el FC.

Estudios previos han reportado resultados similares, particularmente uno realizado con adultos mayores a 50 años, en el cual la presión arterial sistólica, el colesterol y el índice de masa corporal, estuvieron asociados a deterioro cognitivo global $\mathrm{y}$, también con procesos cognitivos específicos como memoria y función ejecutiva ${ }^{34}$. Otro estudio con población europea demostró la asociación entre diabetes, hipertensión y consumo de tabaco con deterioro cognitivo ${ }^{11}$. La hipertensión es un factor que incide en la función cognitiva y puede incrementar el riesgo de tener deterioro cognitivo $\mathrm{y}$ demencia ${ }^{35}$. El colesterol HDL se ha vinculado con la aterosclerosis ${ }^{36}$, una de las afecciones vasculares más comunes que actúa como precursor de deterioro cognitivo y demencia ${ }^{37}$.

En este estudio, y contrario a lo esperado, no se obtuvo evidencia respecto al rol mediador de la $\mathrm{RC}$ en la asociación de los factores de riesgo cardiometabólico y FC, sin embargo, los resultados demuestran asociaciones directas entre las variables estudiadas, y el efecto de mediación fue marginalmente significativo $(p=0,07)$. Diversos estudios han identificado una asociación entre factores de riesgo cardiometabólico como obesidad, síndrome metabólico e hipertensión, y RC, el mecanismo más plausible para explicar esta relación es que la enfermedad vascular genera cambios en la estructura y función del cerebro alterando la reserva cerebral y la $\mathrm{RC}^{14,18,38}$. En cuanto a la RC y el funcionamiento cognitivo, estos resultados coinciden con estudios previos, los cuales han evidenciado el efecto protector de la RC, específicamente, una alta participación en actividades de ocio se asocia con una disminución del riesgo de deterioro cognitivo vascular en adultos mayores ${ }^{39} \mathrm{y}$ la merma del progreso de los síntomas en la $\mathrm{EA}^{40}$.

Este estudio tiene fortalezas y limitaciones. En relación a las primeras, este estudio es innovador por cuanto estudió la asociación entre factores de riesgo cardiometabólico y elementos conductuales medidos a través de la reserva cognitiva, $y$ el funcionamiento cognitivo, en una muestra de 300 sujetos, cuya edad promedio fue menor a 60 años. Esto permitió relevar el rol que los factores de riesgo cardiometabólico tienen en el FC y en la RC en un grupo etario de menor edad de los que típicamente son estudiados. De esta forma, estos resultados contribuyen con evidencia que permitiría desarrollar iniciativas e intervenciones de prevención y promoción en el ámbito clínico, que promuevan conductas saludables, para favorecer la salud cognitiva en población de mediana edad y envejecida.

En relación a las limitaciones, el diseño transversal del estudio no permite identificar relaciones de causalidad entre las variables, pese a que teóricamente, es más probable que los factores de riesgo cardiometabólico antecedan temporalmente al declive del funcionamiento cognitivo. Por otra parte, se sugiere cautela a la hora de generalizar los resultados de este estudio, por cuanto la muestra presenta un promedio de escolaridad relativamente mayor que la media nacional.

Como futuras líneas de investigación, se propone incluir marcadores biológicos de inflamación y factores conductuales como ingesta alimentaria, que permitan abarcar otros indicadores de riesgo cardiometabólico. En relación con la reserva cognitiva se propone explorar el rol de la hipoacusia y consumo de alcohol. Debido a que los adultos pueden experimentar problemas de salud mental, para futuros estudios se sugiere incorporar al modelo variables psicológicas como síntomas depresivos o ansiosos, ya que permitiría una comprensión integral del fenómeno estudiado.

Agradecimientos: Este estudio recibió el apoyo de la Comisión Nacional de Investigación Científica y Tecnológica (CONICYT) del Gobierno de Chile. Se agradece al equipo de estudiantes de pregrado que apoyaron en el proceso de recolección de datos: Ignacia Barros, Constanza Baumert, 
Catalina Becerra, Franchesca Beneventi, Damián Medina y Bárbara Ortiz, y a cada una de las personas que participaron en este estudio.

\section{Referencias}

1. United Nations PDD of E and SA. World Aging Population 2017. United Nations New York. 2017:1-40. doi: 10.1049/el:20000788.

2. Instituto Nacional de Estadísticas. Estimaciones y proyecciones de la población de Chile 1992-2050. Total país. Metodología y principales resultados. 2018:47. http://www.censo2017.cl/descargas/proyecciones/ metodologia-estimaciones-y-proyecciones-de-poblacion-chile-1992-2050.pdf.

3. CELADE. Estimaciones y Proyecciones de Población a Largo Plazo. 1950-2100; 2019.

4. Organización Mundial de la Salud. Informe Mundial Sobre El Envejecimiento y La Salud.; 2015.

5. Cosco TD, Howse K, Brayne C. Healthy ageing, resilience and wellbeing. Epidemiol Psychiatr Sci 2017; 26 (6): 579-83. doi: 10.1017/s2045796017000324.

6. Ribera JM. Dignidad de la persona mayor. Rev Esp Geriatr Gerontol. 2015; 50 (4): 195-9. doi: 10.1016/j. regg.2015.01.003.

7. Meusel L-AC, Anderson ND, Parrott MD, Yuen W, Tchistiakova E, Macintosh BJ, Feldman S, Greenwood CE. Brain Function Is Linked to LDL Cholesterol in Older Adults with Cardiovascular Risk. J Am Geriatr Soc 2017; 65 (2): 51-5.

8. Pieramico V, Esposito R, Cesinaro S, Frazzini V, Sensi SL. Effects of non-pharmacological or pharmacological interventions on cognition and brain plasticity of aging individuals. Front Syst Neurosci. 2014; 8: 1-10. doi: $10.3389 /$ fnsys.2014.00153.

9. Fisher GG, Chaffee DS, Tetrick LE, Davalos DB, Potter GG. Cognitive functioning, aging, and work: A review and recommendations for research and practice. J Occup Health Psychol 2017; 22 (3): 314-36. doi: 10.1037/ ocp0000086.

10. Borrás C, Viña J. Neurofisiología y envejecimiento. Concepto y bases fisiopatológicas del deterioro cognitivo. Rev Esp Geriatr Gerontol 2016; 51 (1): 3-6. doi: 10.1016/ S0211-139X(16)30136-6.

11. Lourenco J, Serrano A, Santos-Silva A, Gomes M, Afonso C, Freitas P, et al. Cardiovascular risk factors are correlated with low cognitive function among older adults across Europe based on the share database. Aging Dis 2018; 9 (1): 90-101. doi: 10.14336/ad.2017.0128.

12. Yaffe K, Vittinghoff E, Pletcher MJ, Hoang TD, Launer
LJ, Whitmer RA, et al. Early adult to midlife cardiovascular risk factors and cognitive function. Circulation 2014; 129 (15): 1560-7. doi: 10.1161/CIRCULATIONAHA. 113.004798

13. Waldstein SR. The relation of hypertension to cognitive function. Curr Dir Psychol Sci 2003; 12 (1): 9-12.

14. Alipour H, Goldust M. The association between blood pressure components and cognitive functions and cognitive reserve. Clin Exp Hypertens 2016; 38 (1): 95-9. doi: 10.3109/10641963.2015.1047946.

15. Camafort M, Sierra C. Hipertensión arterial y demencia: Una relación compleja. Rev Esp Geriatr Gerontol 2016; 51 (1): 3-4. doi: 10.1016/j.regg.2015.09.018.

16. Gorelick PB, Nyenhuis D. Understanding and treating vascular cognitive impairment. Continuum (NY) 2013; 19 (2): 425-37. doi: 10.1212/01.CON.0000429174.29601. de.

17. Toro P, Degen C, Pierer M, Gustafson D, Schröder J, Schönknecht P. Cholesterol in mild cognitive impairment and Alzheimer's disease in a birth cohort over 14 years. Eur Arch Psychiatry Clin Neurosci 2014; 264 (6): 485-92. doi: 10.1007/s00406-013-0468-2.

18. Ihle A, Gouveia ÉR, Gouveia BR, Freitas DL, Tinôco MA, Kliegel M . High-Density Lipoprotein Cholesterol Level Relates to Working Memory, Immediate and Delayed Cued Recall in Brazilian Older Adults: The Role of Cognitive Reserve. Dement Geriatr Cogn Disord 2017; 44 (1-2): 84-91. doi: 10.1159/000477846.

19. Singh-Manoux A, Gimeno D, Kivimaki M, Brunner E, Marmot MG. Low hdl cholesterol is a risk factor for deficit and decline in memory in midlife. The whitehall II study. Arterioscler Thromb Vasc Biol. 2008; 28 (8): 1556-62.

20. van den Kommer TN, Dik MG, Comijs HC, Jonker C, Deeg DJH. The role of lipoproteins and inflammation in cognitive decline: Do they interact? Neurobiol Aging. 2012; 33 (1): 196.e1-196.e12. doi: 10.1016/j.neurobiolaging.2010.05.024

21. Arteaga A, Maiz A, Rigotti A, Cortés V. Asociación entre diabetes mellitus y patología cardiovascular en la población adulta de Chile: Estudio de la Encuesta Nacional de Salud 2009-2010. Rev Med Chile 2014; 142 (2): 175-83. doi: 10.4067/S0034-98872014000200005.

22. Cheng G, Huang C, Deng H, Wang H. Diabetes as a risk factor for dementia and mild cognitive impairment: a meta-analysis of longitudinal studies. Intern Med J 2012; 42 (5): 484-91. doi: 10.1111/j.1445-5994.2012.02758.x.

23. Zhao Q, Roberts RO, Ding D, Cha R, Qihao G, Meng $\mathrm{H}$, et al. Diabetes is associated with worse executive function in both eastern and western populations: Shanghai aging study and mayo clinic study of aging. J 
Alzheimer's Dis 2015; 47 (1): 167-76. doi: 10.3233/jad150073.

24. Atti AR, Forlani C, De Ronchi D, Palmer K, Casadio P, Dalmonte E, et al. Cognitive impairment after age 60: Clinical and social correlates in the "faenza Project." J Alzheimer's Dis 2010; 21 (4): 1325-34. doi: 10.3233/ JAD-2010-091618.

25. John A, Desai R, Richards M, Gaysina D, Stott J. Role of cardiometabolic risk in the association between accumulation of affective symptoms across adulthood and mid-life cognitive function: national cohort study. Br J Psychiatry. July 2020: 1-7. doi: 10.1192/bjp.2020.123

26. Alkan E, Taporoski TP, Sterr A, von Schantz M, Vallada $\mathrm{H}$, Krieger JE, et al. Metabolic syndrome alters relationships between cardiometabolic variables, cognition and white matter hyperintensity load. Sci Rep 2019; 9 (1): 1-9. doi: 10.1038/s41598-019-40630-6.

27. Stern Y, Arenaza-Urquijo EM, Bartrés-Faz D, Belleville S, Cantilon M, Chetelat G, et al. Whitepaper: Defining and investigating cognitive reserve, brain reserve, and brain maintenance. Alzheimer's Dement. 2018; (September): 1-7. doi: 10.1016/j.jalz.2018.07.219.

28. Lavrencic LM, Richardson C, Harrison SL, Muniz-Terrera $\mathrm{G}$, Keage $\mathrm{HAD}$, Brittain $\mathrm{K}$, et al. Is there a link between cognitive reserve and cognitive function in the oldest-old? Journals Gerontol - Ser A Biol Sci Med Sci 2018; 73 (4): 499-505. doi: 10.1093/gerona/glx140.

29. Cancino M, Rehbein L, Ortiz MS. Funcionamiento cognitivo en adultos mayores: rol de la reserva cognitiva, apoyo social y depresión. Rev Med Chile 2018; 146 (3): 315-22. doi: 10.4067/s0034-98872018000300315.

30. Giordano N, Tikhonoff V, Palatini P, Bascelli A, Boschetti G, De Lazzari F, et al. Cognitive functions and cognitive reserve in relation to blood pressure components in a population-based cohort aged 53 to 94 years. Int J Hypertens 2012; 2012: 1-8. doi: 10.1155/2012/274851.

31. Ihle A, Gouveia ÉR, Gouveia BR, Freitas DL, Jurema J, Machado FT, et al. The relation of hypertension to performance in immediate and delayed cued recall and working memory in old age: The role of cogni- tive reserve. J Aging Health 2018; 30 (8): 1171-87. doi: 10.1177/0898264317708883.

32. Matias-Guiu JA, Fernández de Bobadilla R, Escudero G, Pérez-Pérez J, Cortés A, Morenas-Rodríguez E, et al. Validación de la versión española del test Addenbroke's Cognitive Examination III para el diagnóstico de demencia. Neurología 2014; 30 (9): 529-94. doi: 10.1016/j. nrl.2014.05.004.

33. León I, García J, Roldán-Tapia L. Construcción de la escala de reserva cognitiva en población española: estudio piloto. Rev Neurol 2011; 52 (11): 653-60.

34. Dregan A, Stewart R, Gulliford MC. Cardiovascular risk factors and cognitive decline in adults aged 50 and over: A population-based cohort study. Age Ageing. 2013;42(3):338-345. doi: 10.1093/ageing/afs 166

35. Walker KA, Power MC, Gottesman RF. Defining the relationship between hypertension, cognitive decline, and dementia: a review. Curr Hypertens Rep 2018; 19 (3): 1-27. doi: 10.1007/s11906-017-0724-3.Defining.

36. Parhofer KG. Increasing HDL-cholesterol and prevention of atherosclerosis: A critical perspective. Atheroscler Suppl 2015; 18: 109-11. doi: 10.1016/j.atherosclerosissup.2015.02.020.

37. Chen WH, Jin W, Lyu PY, Liu Y, Li R, Hu M, et al. Carotid atherosclerosis and cognitive impairment in nonstroke patients. Chin Med J (Engl). 2017; 130 (19): 2375-9. doi: 10.4103/0366-6999.215331.

38. Miu J, Negin J, Salinas-Rodríguez A, Manrique-Espinoza B, Sosa-Ortiz AL, Cumming R, et al. Factors associated with cognitive function in older adults in Mexico. Glob Health Action. 2016; 9 (1): 30747. doi: 10.3402/ gha.v9.30747.

39. Verghese J, Wang C, Katz MJ, Sanders A, Lipton RB. Leisure activities and risk of Vascular Cognitive Impairment in older adults. J Geriatr Psychiatry Neurol. 2009; 22 (2): 110-8. doi: 10.1371/journal.pone.0178059.

40. van Loenhoud AC, van der Flier WM, Wink AM, Dicks E, Twisk J, Barkhof F, et al. Cognitive reserve and clinical progression in Alzheimer disease. Neurology 2019; 93: 334-46. doi: 10.1212/WNL.0000000000007821. 